\title{
Acute akinetic-rigid syndrome in COVID-19 encephalitis
}

\author{
Boby Varkey Maramattom $^{1}\left[\mathbb{D}^{\cdot}\right.$. Asha Kishore ${ }^{1}$
}

Received: 24 November 2021 / Accepted: 3 February 2022 / Published online: 22 February 2022

(C) The Author(s) under exclusive licence to Belgian Neurological Society 2022

Keywords Acute akinetic-rigid syndrome $\cdot$ COVID-19 encephalitis $\cdot$ Acute akinetic-rigid syndrome $\cdot$ COVID-19 encephalitis · Akinetic rigid syndrome · COVID-19 encephalitis · Akinetic rigid syndrome · COVID-19

Sir,

Both Parkinsonism and akinetic mutism (AM) have been described in association with COVID-19. Parkinsonism cases had evidence of nigral degeneration in DAT scans or hypoxic damage to the basal ganglia [1,2]. The majority recovered spontaneously or responded to dopamine agonist therapy. One case of a hypokinetic- rigid syndrome has been reported with COVID-19 [3]. However, this patient had asymmetrical presynaptic nigrostriatal pathway impairment on DaT-SPECT and showed significant improvement in the clinical picture without any specific treatment. Cases with AM associated with COVID-19 due to the disruption of cortico-striato-thalamo-cortical circuits originating in the anterior cingulate cortex (ACC) are also known [4]. We report a COVID-19 encephalitis presenting with an acute akinetic- rigid syndrome.

A 75-year-old man, previously in good health, returned from farm work on the day of the illness and collapsed in his bed. When aroused, a few minutes later, he was found to be febrile and confused. On arrival at the hospital, he was febrile, comatose, with persistent downgaze, severe generalized rigidity and bilateral extensor plantar responses. He was intubated for airway protection. RT-PCR for SARS- CoV-2 was positive. There was no history of exposure to toxins or neuroleptics.

Blood reports on day 2 were notable for elevated Troponin I $(353.3 \mathrm{ng} / \mathrm{L}-$ normal < 13) and d-dimer $(1500 \mathrm{ng} /$ $\mathrm{ml}$ ). His CSF was abnormal (total count 90 , lymphocytes $89 \%$, neutrophils $6 \%$, monocytes $4 \%$, protein $-165 \mathrm{mg} / \mathrm{dl}$ ) and showed elevated CSF antibody to SARS CoV2 spike

Boby Varkey Maramattom

bobvarkey@gmail.com

1 Centre of Excellence in Neurosciences, Aster Medcity, Kochi, Kerala 682027, India protein (> $250 \mathrm{U} / \mathrm{ml}$ ). RT-PCR for SARS-CoV-2 in CSF was negative. An encephalitic multiplex PCR panel (Streptococcus pneumoniae, Haemophilus influenzae, Neisseria meningitidis, Mycobacterium tuberculosis, Cryptococcus neoformans, Herpes Simplex Virus 1 \& 2, Cytomegalovirus, Varicella Zoster Virus, Human Herpes Virus-6, John Cunningham Virus, JEV, Dengue 1-4, West Nile, Enteroviruses, Chikungunya, Rabies, Chandipura, Measles, Mumps, Rubella, Nipah virus and Toxoplasma gondii) was negative. A neuronal autoimmune encephalitis panel was also negative. MRI brain showed FLAIR hyperintensity in the right frontal region with overlying leptomeningeal enhancement and small, punctate, gradient-blooming lesions in the right hemisphere [micro-hemorrhages]. (Figure) By day three, he was conscious but had an acute akinetic-rigid syndrome with corticospinal tract involvement and mutism. Dexamethasone ( $8 \mathrm{mg} /$ day) and Remdesivir were started along with a trial of Levodopa/Carbidopa (100/25 mg) QID which was increased to $250 / 62.5 \mathrm{mg}$ QID by day six (total of $1000 / 250 \mathrm{mg}$ ). He underwent tracheostomy on day seven and was shifted to the ward on day ten.

${ }^{18}$ F-FDOPA scan on day ten, showed normal tracer uptake in the basal ganglia. ${ }^{18} \mathrm{FDG}$ PET/CT revealed reduced FDG uptake in bilateral frontal cortices. FDG uptake was normal in the rest of the brain, including the basal ganglia (Fig. 1).

Levodopa/Carbidopa 1000/250 mg was continued for 2 weeks and was tapered after one month to Levodopa/Carbidopa 300/75 mg daily. His clinical examination remained stable. At follow up, two months later, however, he was ambulant, but apathetic and had mild cognitive impairment, mild residual rigidity and bradykinesia. At follow up, four months later, he had nearly attained his baseline level of functioning (mRS score 1$)$.

We report a case of COVID-19 encephalitis that caused an acute akinetic-rigid syndrome [1]. The clinico-radiological picture suggested involvement of the fronto-striatal 
Fig. 1 Panel A; Axial FLAIR MRI image showing a right frontal hyperintensity. Panel B; Axial gradient echo image showing punctate right hemispheric hemorrhages. Panel C; Axial post contrast $\mathrm{T} 1$ weighted image showing right frontal cortical/leptomeningeal enhancement. Panel D; ${ }^{18}$ F-FDOPA scan showing normal basal ganglia uptake. Panel E; Axial

${ }^{18}$ FDG PET/CT image showing diffuse fronto-parietal hypometabolism

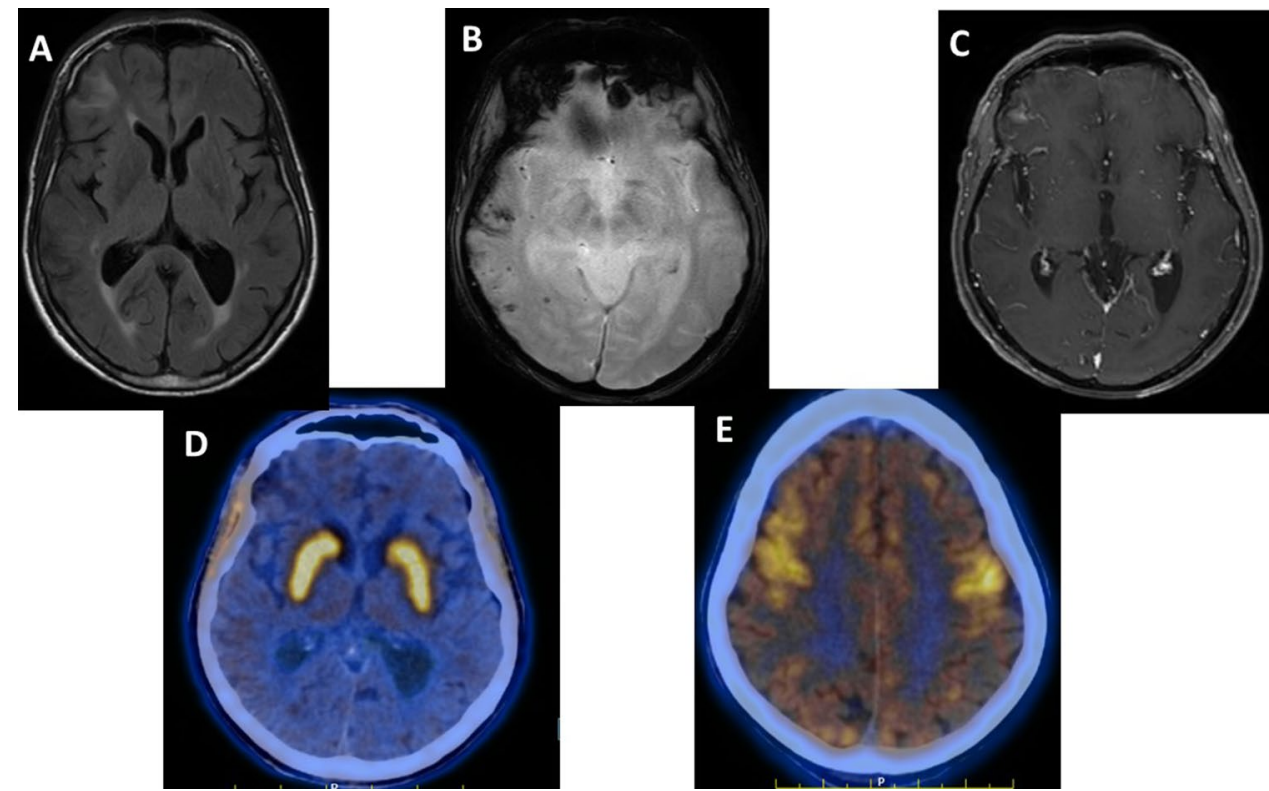

circuits (abnormal frontal hypometabolism in FDG PET/CT) with sparing of the nigrostriatal system (normal ${ }^{18} \mathrm{~F}$-FDOPA scan). CSF Pleocytosis and focal MRI brain abnormalities were compatible with COVID-19 encephalitis, with the exclusion of other causes.

A case of transient steroid-responsive encephalopathy presenting as akinetic mutism has been reported, however our case had a more prolonged course, and unresponsiveness to steroid therapy as well as fronto-parietal hypometabolism on FDG PET CT implicating the frontal-subcortical circuitry. In comparison with the case of an acute hypokineticrigid syndrome following SARS-CoV-2 infection which showed nigrostrial involvement, our patient had a normal nigrostriatal function on 18F-FDOPA scans [3,5]. The mutism in our patient further implicates the medial forebrain region, especially the anterior cingulate cortex (ACC) and its connections with diverse brain regions including the cingulate motor areas and the cortico-striato-thalamo-cortical loops.

SARS-CoV-2 enters the brain by hematogenous route, direct invasion through vascular pericytes, via axoplasmic spread or retrograde trans-cribriform spread. In COVID-19 encephalitis, CSF examination discloses mild pleocytosis but CSF RT-PCR is usually negative. Oligoclonal bands and elevated levels of serum and CSF IL- 8 and TNF- $\alpha$ concentrations, glial fibrillary acidic protein and neurofilament light chain (nFL) are reported in CSF. Most of the CNS injury in COVID-19 encephalitis is attributed to a dysregulated host response (hyperinflammatory immune response) rather than a direct viral cytopathic effect.

We were unable to detect SARS CoV-2 in the CSF by PCR or obtain a brain biopsy to rule out a direct viral neuronal cytopathy. Nevertheless, the presence of intrathecal anti-spike antibodies suggested an immune mediated encephalitis, which involved the cortical supplementary motor circuits and manifested as a severe akinetic- rigid syndrome. Our patient made a very slow recovery over 4 months, suggestive of a gradual improvement in the dysfunctional cortical-subcortical circuitry.

\section{Declarations}

Conflict of interest The authors declare that they have no conflict of interest.

Ethical approval This article does not contain any studies with human participants or animals performed by any of the authors.

Informed consent For this type of study formal consent is not required.

\section{References}

1. Fearon C, Mikulis DJ, Lang AE (2021) Parkinsonism as a sequela of SARS-CoV-2 infection: pure hypoxic injury or additional COVID-19-related response? Mov Disord 36(7):1483-1484

2. Faber I, Brandão PRP, Menegatti F, CarvalhoBispo DD, Maluf FB, Cardoso F (2020) Coronavirus disease 2019 and parkinsonism: a non-post-encephalitic case. Mov Disord. 35(10):1721-1722

3. Méndez-Guerrero A, Laespada-García MI, Gómez-Grande A, Ruiz-Ortiz M, Blanco-Palmero VA, Azcarate-Diaz FJ et al (2020) Acute hypokinetic-rigid syndrome following SARSCoV-2 infection. Neurology 95(15):e2109-e2118 
4. Fusunyan M, Praschan N, Fricchione G, Beach S (2021) Akinetic mutism and coronavirus disease 2019: a narrative review. J Acad Consult Psychiatry 62(6):625

5. Chen S, Han Y, Yang L, Kim T, Nair M, Harschnitz O et al (2021) SARS-CoV-2 infection causes dopaminergic neuron senescence. Res Sq. 21:rs.3.rs-513461
Publisher's Note Springer Nature remains neutral with regard to jurisdictional claims in published maps and institutional affiliations. 